\title{
SEISMIC WAVES IN A RANDOMLY STRATIFIED EARTH MODEL
}

\author{
Richard BOURRET \\ Venezuelan Foundation for Seismological Research, Funvisis, \\ Apartado Postal 1892, Caracas, Venezuela
}

(Received June 30, 1980)

Stochastic perturbation theory is used to study the propagation of plane seismic waves in a medium in which random fluctuations in local density and elastic shear modulus are considered. Random horizontal stratification is modeled by the use of anisotropic correlation functions for these variables. The results indicate that the $\mathrm{P}$-wave ( $\mathrm{S}$-wave) group velocity is increased (decreased) when $k l .1$; the oppossite occurs when $k l<1$. The changes for the phase velocities are in the opposite sense. Attenuation coefficients are given for the P, SH and SV components. The results agree with a calculation (BourRET, 1980) using the parabolic approximation (valid only for $k l>1$ ).

\section{Introduction}

If we write the density and elastic shear modulus as

$$
\begin{aligned}
& \rho=\bar{\rho}(1+\delta(\boldsymbol{r})), \\
& \mu=\mu(1+\varepsilon(\boldsymbol{r})),
\end{aligned}
$$

where $\delta$ and $\varepsilon$ are the random fractional fluctuations of $\rho$ and $\mu$, then it is straightforward to show, making the additional assumption

$$
\frac{\partial \varepsilon}{\partial x_{3}} \gg \frac{\partial \varepsilon}{\partial x_{1}}, \frac{\partial \varepsilon}{\partial x_{2}}
$$

that the elastodynamic equations take the form

$$
\begin{aligned}
& \mathrm{d}^{2} P \\
& \mathrm{~d} x_{1}{ }^{2}+k_{\mathrm{p}}{ }^{2} P=k_{\mathrm{p}}{ }^{2} \lambda P-\frac{1}{3} \varepsilon_{3} \frac{\mathrm{d} S}{\mathrm{~d} x_{1}}, \\
& \mathrm{~d}^{2} H \\
& \mathrm{~d} x_{1}{ }^{2}+k_{\mathrm{s}}{ }^{2} H=k_{\mathrm{s}}{ }^{2} \lambda H, \\
& \mathrm{~d}^{2} S \\
& \mathrm{~d} x_{1}{ }^{2}+k_{\mathrm{n}}{ }^{2} S=k_{\mathrm{g}}{ }^{2} \lambda S-\varepsilon, \frac{\mathrm{d} P}{\mathrm{~d} x_{1}},
\end{aligned}
$$

in which $(P, H, S)$ are the displacements associated with the $\mathrm{P}, \mathrm{SH}$, and $\mathrm{SV}$ waves. We have also written, for brevity, 


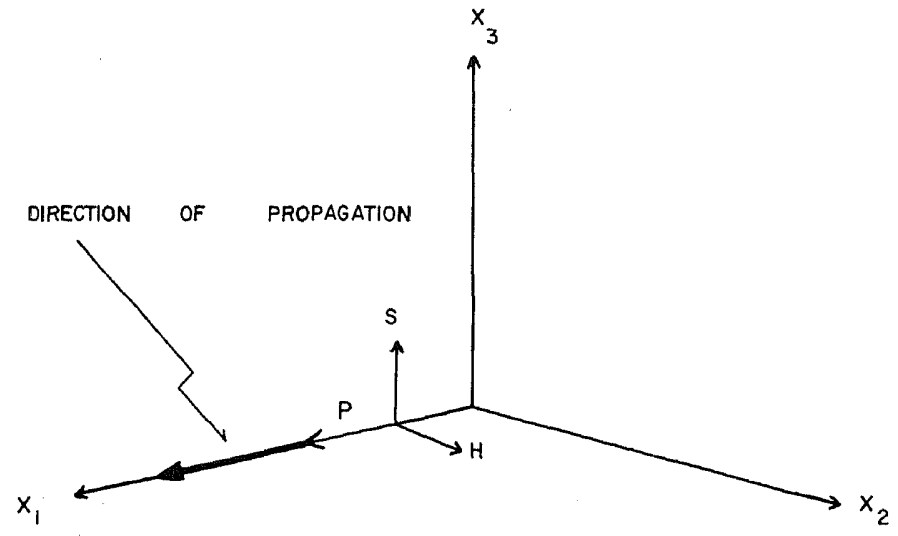

Fig. 1

$$
\begin{aligned}
& \lambda \equiv \delta-\varepsilon, \\
& \varepsilon_{, 3}=\frac{\partial \varepsilon}{\partial x_{3}} .
\end{aligned}
$$

The values of $\bar{\rho}$ and $\bar{\mu}$ have been assimilated into

$$
k_{\mathrm{p}}^{2}=3 \bar{\mu} / \bar{\rho}, \quad k_{\mathrm{s}}{ }^{2}=\bar{\mu} / \bar{\rho} .
$$

By neglecting horizontal gradients of $\mu$ in comparison with the vertical gradient, we have taken a model in which the stratification is horizontal. (Derivatives of $\rho$ do not occur.) And by limiting the coordinate dependence to $x_{1}$, we have chosen the wave propagation in this direction. The situation is shown in the figure.

\section{Calculation of the Coherent Wave}

The calculation of the coherent, or ensemble mean, wave is accomplished by means of random perturbation theory. The theory is given in BourRET (1962, 1965), FRISCH (1968) and more recently in HASAN (1973).

We shall set Eq. (1.5) for $H$ (i.e., $\mathrm{SH}$ ) aside, temporarily, since it is not coupled to the other equations of $\mathrm{P}$ and SV. The results for (1.5) can be written down intuitively from the analysis of $\mathrm{P}$ and $\mathrm{SV}$ as we shall see. These coupled equations are

$$
\begin{aligned}
& \left(\frac{\mathrm{d}^{2}}{\mathrm{~d} x_{1}^{2}}+k_{\mathrm{p}}{ }^{2}\right) P=k_{\mathrm{p}}{ }^{2} \lambda P-\frac{1}{3} \varepsilon, \frac{\mathrm{d} S}{\mathrm{~d} x_{1}}, \\
& \left(\frac{\mathrm{d}^{2}}{\mathrm{~d} x_{1}^{2}}+k_{\mathrm{s}}{ }^{2}\right) S=k_{\mathrm{s}}{ }^{2} \lambda S-\varepsilon, 3 \frac{\mathrm{d} P}{\mathrm{~d} x_{1}},
\end{aligned}
$$

and the Green's functions in one-dimensional free space for the $P$ and $S$ functions are 


$$
\begin{aligned}
& G_{\mathrm{p}}\left(x_{1}\right)=\frac{1}{2 i k_{\mathrm{p}}} \exp \left\{i k_{\mathrm{p}}\left|x_{1}\right|\right\} \\
& G_{\mathrm{s}}\left(x_{1}\right)=\frac{1}{2 i k_{\mathrm{s}}} \exp \left\{i k_{\mathrm{s}}\left|x_{1}\right|\right\} .
\end{aligned}
$$

The $P$ and $S$ appearing on the left-hand-sides can be obtained in terms of the material on the right-hand-side by means of these Green's functions as follows:

$$
\begin{aligned}
& P(x)=k_{\mathrm{g}}{ }^{2} \int_{0}^{\infty} \lambda\left(r_{1}\right) G_{\mathrm{p}}\left(x-x_{1}\right) P\left(x_{1}\right) \mathrm{d} x_{1}-\frac{1}{3} \int_{0}^{\infty} \varepsilon_{3}\left(r_{1}\right) G_{\mathrm{p}}\left(x-x_{1}\right) S,{ }_{1}\left(x_{1}\right) \mathrm{d} x_{1}, \\
& S(x)=k_{\mathrm{s}}{ }^{2} \int_{0}^{\infty} \lambda\left(r_{1}\right) G_{\mathrm{s}}\left(x-x_{1}\right) S\left(x_{1}\right) \mathrm{d} x_{1}-\int_{0}^{\infty} \varepsilon,,_{3}\left(r_{1}\right) G_{\mathrm{s}}\left(x-x_{1}\right) P_{, 1}\left(x_{1}\right) \mathrm{d} x_{1} .
\end{aligned}
$$

We shall also need the expressions for $\mathrm{d} S / \mathrm{d} x$ and $\mathrm{d} P / \mathrm{d} x$ which are obtained directly from the foregoing;

$$
\begin{aligned}
& \frac{\mathrm{d} P}{\mathrm{~d} x_{1}}=k_{\mathrm{r}}^{2} \int_{11}^{\infty} \lambda\left(r_{1}\right) G_{\mathrm{P}_{1}}\left(x-x_{1}\right) P\left(x_{1}\right) \mathrm{d} x_{1}-\frac{1}{3} \int_{0}^{\infty} \varepsilon,_{3}\left(r_{1}\right) G_{\mathrm{P}_{1}}\left(x-x_{1}\right) S_{11}\left(x_{1}\right) \mathrm{d} x_{1}, \\
& \frac{\mathrm{d} S}{\mathrm{~d} x_{1}}=k_{*}{ }^{2} \int_{0}^{\infty+\infty} \lambda\left(r_{1}\right) G_{\mathrm{S}_{1}}\left(x-x_{1}\right) S\left(x_{1}\right) \mathrm{d} x_{1}-\int_{0}^{\infty} \varepsilon_{, 3}\left(r_{1}\right) G_{\mathrm{S}_{1}}\left(x-x_{1}\right) P_{,_{1}}\left(x_{1}\right) \mathrm{d} x_{1} .
\end{aligned}
$$

We now introduce these last four expressions into the right-hand terms of (2.1) and (2.2), take the ensemble averages, and obtain the following integro-differential equations for the coherent fields (after a shift of variables on the right-hand-side).

$$
\begin{aligned}
\left(\frac{\mathrm{d}^{2}}{\mathrm{~d} x_{1}{ }^{2}}+k_{\mathrm{p}}{ }^{2}\right)\left\langle P\left(x_{1}\right)\right\rangle= & \left\langle\lambda^{2}\right\rangle k_{\mathrm{p}}{ }^{4} \int_{0}^{\infty} \Gamma(y) G_{\mathrm{y}}(y)\langle P(x-y)\rangle \mathrm{d} y \\
& +\frac{1}{3} l_{3}{ }^{-2}\left\langle\varepsilon^{2}\right\rangle \int_{0}^{\infty} \Gamma,_{3,3}(y) G_{\mathrm{s}_{1}}(y)\left\langle P,,_{1}(x-y)\right\rangle \mathrm{d} y, \\
\left(\frac{\mathrm{d}^{2}}{\mathrm{~d} x_{1}{ }^{2}}+k_{\mathrm{s}}{ }^{2}\right)\left\langle S\left(x_{1}\right)\right\rangle= & \left\langle\lambda^{2}\right\rangle k_{\mathrm{s}}{ }^{4} \int_{0}^{\infty} \Gamma(y) G_{\mathrm{s}}(y)\langle S(x-y)\rangle \mathrm{d} y \\
& +\frac{1}{3} l_{3} l^{-2}\left\langle\varepsilon^{2}\right\rangle \int_{0}^{\infty} \Gamma{ }_{, 3,3}(y) G_{\mathrm{P}_{1}}(y)\langle S(x-y)\rangle \mathrm{d} y .
\end{aligned}
$$

The $\Gamma$ symbols for autocorrelations are described in detail in the appendix. The commas indicate differentiation with respect to the numbered variable $(1,2,3) \leftrightarrow$ $\left(x_{1}, x_{2}, x_{3}\right)$. From the form of (1.5) for $H$ it is apparent that the equation for $H$ is

$$
\left(\frac{\mathrm{d}^{2}}{\mathrm{~d} x_{1}{ }^{2}}+k_{\mathrm{s}}{ }^{2}\right)\langle H(x)\rangle=\left\langle\lambda^{2}\right\rangle k_{\mathrm{s}}{ }^{4} \int_{0}^{\infty} \Gamma(y) G_{\mathrm{H}}(y)\langle H(x-y)\rangle \mathrm{d} y .
$$

Now in Eqs. (2.9), (2.10) and (2.11) the unperturbed wave numbers are defined by

$$
k_{\mathrm{p}}={\stackrel{(1)}{v_{\mathrm{p}}}}^{(1)}, k_{\mathrm{s}}=\frac{(\omega)}{v_{\mathrm{s}}},
$$


while we shall write the perturbed values in capital letters corresponding to the perturbed or primed values of the new phase velocities, thus:

$$
K_{\mathrm{p}}=\frac{\omega}{v_{\mathrm{p}}{ }^{\prime}}, \quad K_{\mathrm{s}}=\frac{\omega}{v_{\mathrm{s}}{ }^{\prime}} .
$$

We shall introduce into the aforementioned equations the trial solutions

$$
\langle P(x)\rangle=\mathrm{e}^{i K_{\mathrm{p}} x} \quad\langle S(x)\rangle=\mathrm{e}^{i K_{\mathrm{B}} x} .
$$

Knowing the Green's functions (2.3) and (2.4), and the autocorrelation functions, Appendix I, we can evaluate the equations (2.7) and (2.8). We obtain an expression which we shall write symbolically as

$$
\begin{aligned}
& -K_{\mathrm{p}}{ }^{2}+k_{\mathrm{p}}{ }^{2}=F\left(K_{\mathrm{p}}, k_{\mathrm{p}}, k_{\mathrm{s}}\right), \\
& -K_{\mathrm{s}}{ }^{2}+{k_{\mathrm{s}}}^{2}=G\left(K_{\mathrm{s}}, k_{\mathrm{p}}, k_{\mathrm{s}}\right) .
\end{aligned}
$$

On the right-hand-side we make the approximation

$$
K_{\mathrm{p}} \approx k_{\mathrm{p}}, \quad K_{\mathrm{s}} \approx k_{\mathrm{s}} \text {. }
$$

Rewriting the expressions on the left into factors we obtain

$$
\begin{aligned}
& \left(-K_{\mathrm{p}}+k_{\mathrm{p}}\right)\left(K_{\mathrm{p}}+k_{\mathrm{p}}\right)=F\left(k_{\mathrm{p}}, k_{\mathrm{p}}, k_{\mathrm{s}}\right), \\
& \left(-K_{\mathrm{s}}+k_{\mathrm{s}}\right)\left(K_{\mathrm{s}}+k_{\mathrm{s}}\right)=G\left(k_{\mathrm{s}}, k_{\mathrm{p}}, k_{\mathrm{s}}\right) .
\end{aligned}
$$

Writing again $K_{\mathrm{p}} \approx k_{\mathrm{p}}$ and $K_{\mathrm{s}} \approx k_{\mathrm{s}}$ in each of the second parenthesis on the left gives, after a simple rearrangement

$$
\begin{aligned}
& K_{\mathrm{p}}=k_{\mathrm{p}}-\frac{1}{2 k_{\mathrm{p}}} F\left(k_{\mathrm{p}}, k_{\mathrm{p}}, k_{\mathrm{s}}\right), \\
& K_{\mathrm{s}}=k_{\mathrm{s}}-\frac{1}{2 k_{\mathrm{s}}} G\left(k_{\mathrm{s}}, k_{\mathrm{p}}, k_{\mathrm{s}}\right) .
\end{aligned}
$$

The expressions $F$ and $G$ are complex. Writing out (2.20) and (2.21) explicitly with real and imaginary parts separated yields:

$$
\begin{aligned}
& K_{\mathrm{p}}=\left[k_{\mathrm{p}}-\frac{\left\langle\varepsilon^{2}\right\rangle k S^{2}}{12\left(1+\left(k l_{1}\right)^{2}\right)}\right]+i\left[\frac{\left\langle\lambda^{2}\right\rangle\left(k_{\mathrm{p}} l_{1}\right)^{2}}{2 l_{1}}+\frac{\left\langle\varepsilon^{2}\right\rangle S^{2}}{12 l_{1}\left(1+\left(k l_{1}\right)^{2}\right)}\right], \\
& K_{\mathrm{s}}=\left[k_{\mathrm{s}}+\frac{\left\langle\varepsilon^{2}\right\rangle k S^{2}}{12\left(1+\left(k l_{1}\right)^{2}\right)}\right]+i\left[\frac{\left\langle\lambda^{2}\right\rangle\left(k_{\mathrm{s}} l_{1}\right)^{2}}{2 l_{1}}+\frac{\left\langle\varepsilon^{2}\right\rangle S^{2}}{12 l_{1}\left(1+\left(k l_{1}\right)^{2}\right)}\right],
\end{aligned}
$$

where the following abbreviations have been used:

$$
\begin{gathered}
k \equiv k_{\mathrm{s}}-k_{\mathrm{p}}=(\sqrt{3}-1) k_{\mathrm{p}}, \\
S \equiv l_{1} / l_{3} \gg 1 .
\end{gathered}
$$

From a comparison of Eq. (2.11) for $H$ with the two previous equations it is clear that its dispersion relation must be

$$
K_{\mathrm{sh}}=k_{\mathrm{s}}+i \frac{\left\langle\lambda^{2}\right\rangle\left(k l_{1}\right)^{2}}{2 l_{1}} .
$$




\section{Discussion}

From the above formulas we see easily that the uncoupled SH mode experiences only attenuation as a result of the inhomogeneities. The P and SV modes, however, undergo attenuation and velocity dispersion, and, in particular, the P-mode decreases while the SV mode increases in phase velocity.

Given the approximations made (notably (2.17), it is interesting to observe that our results (2.22) and (2.23) are identical to the results obtained in the above mentioned study (BOURRET, 1980) which uses the parabolic approximation. This means that the full-wave treatment (hyperbolic wave equation) coincides with the parabolic approximation for $k l>1$.

From the expressions (2.22) and (2.23) one can derive (as we did in Bourret, 1980) the group velocities, $U_{\mathrm{m}}, U_{\mathrm{s}}$; we should remark that, in contradistinction to the phase velocities, the S-group velocity is increased and the P-group velocity decreased.

$$
\begin{aligned}
& \frac{1}{U_{\mathrm{p}}}=\frac{1}{v_{\mathrm{p}}}\left\{1-\frac{\left\langle\varepsilon^{2}\right\rangle S^{2}(\sqrt{ } 3-1)}{12}\left[\frac{1-(\sqrt{3}-1)^{2}\left(\omega / v_{\mathrm{p}}\right)^{2} l_{1}^{2}}{\left(1+(\sqrt{3}-1)^{2}\left(\omega / v_{\mathrm{p}}\right) l_{1}\right)^{2}}\right]\right\}, \\
& \frac{1}{U_{\mathrm{H}}}=\frac{1}{v_{\mathrm{s}}}\left\{1+\frac{\left\langle\varepsilon^{2}\right\rangle S^{2}(\sqrt{ } 3-1)}{12}\left[\frac{1-(\sqrt{3}-1)^{2}\left(\omega / v_{\mathrm{p}}\right)^{2} l_{1}^{2}}{\left(1-(\sqrt{3}-1)^{2}\left(\omega / v_{\mathrm{p}}\right)^{2} l_{1}^{2}\right)^{2}}\right]\right\} .
\end{aligned}
$$

Provided $(\sqrt{ } 3-1)\left(\omega / v_{\mathrm{p}}\right) l_{1}>1$; in the case of the opposite inequality $S$ is slowed down and $P$ accelerated.

\section{APPENDIX I}

The cross-correlation functions are obtained by assuming the general form

$$
\Gamma(R)=(1+R) \mathrm{e}^{-R},
$$

which combines simplicity, physical plausibility and has finite derivatives at the origin. Not many other simple functions have these desirable properties. See the discussion in BOURRET (1980)

$$
R^{2}=\left(\begin{array}{c}
x_{1} \\
l_{1}
\end{array}\right)^{2}+\left(\frac{x_{2}}{l_{1}}\right)^{2}+\left(\begin{array}{c}
x_{3} \\
l_{3}
\end{array}\right)^{2}
$$

which leads to

$$
\begin{gathered}
\left\langle\lambda(\boldsymbol{r}) \lambda\left(\boldsymbol{r}^{\prime}\right)\right\rangle=\left\langle\lambda^{2}\right\rangle \Gamma(R), \\
\left\langle\varepsilon_{3}(\boldsymbol{r}) \lambda\left(\boldsymbol{r}^{\prime}\right)\right\rangle=-\langle\varepsilon \lambda\rangle \alpha_{3}{ }^{2} x_{3} \mathrm{e}^{-R}, \\
\left\langle\varepsilon_{3}(\boldsymbol{r}) \varepsilon,{ }_{3}\left(\boldsymbol{r}^{\prime}\right)\right\rangle=-\left\langle\varepsilon^{2}\right\rangle \alpha_{3}{ }^{2} \mathrm{e}^{-\varepsilon_{1}\left(x-x_{1}{ }^{\prime}\right.} ; x_{2}=x_{2}{ }^{\prime} ; x_{3}=x_{3}{ }^{\prime} .
\end{gathered}
$$

Note that

$$
R\left(\boldsymbol{r}-\boldsymbol{r}_{1}{ }^{\prime}\right)=\left[\left(\frac{x_{1}-x_{1}{ }^{\prime}}{l_{1}}\right)^{2}+\left(\frac{x_{2}-x_{2}{ }^{\prime}}{l_{1}}\right)^{2}+\left(\frac{x_{3}-x_{3}{ }^{\prime}}{l_{3}}\right)^{2}\right]^{1 / 2}
$$




$$
\begin{aligned}
& \left\langle\lambda(\boldsymbol{r}) \varepsilon,,_{3}\left(\boldsymbol{r}^{\prime}\right)\right\rangle=0 ; \quad x_{2}=x_{2}{ }^{\prime} ; x_{3}=x_{3}{ }^{\prime}, \\
& \left\langle\varepsilon,,_{3}(\boldsymbol{r}) \lambda\left(\boldsymbol{r}^{\prime}\right)\right\rangle=0 ; \quad x_{2}=x_{2}{ }^{\prime} ; x_{3}=x_{3}{ }^{\prime} .
\end{aligned}
$$

The results (A.4)-(A.7) follow by carrying out the indicated operations using the function defined by (A.1) and (A.2). Note that, unlike the wave variables, $\lambda$ and $\varepsilon$ are dependent upon all three coordinates, but that these coordinates vanish when one arrives at Eqs. (2.10) and (2.11). First consider Eq. (2.9): one obtains for the first term on the right-hand-side

$$
\begin{aligned}
& \left\langle k_{\mathrm{p}}{ }^{2} \lambda(r)\left[k_{\mathrm{p}}{ }^{2} \int_{0}^{\infty} \lambda\left(r_{1}\right) G_{\mathrm{p}}\left(x-x_{1}\right) P\left(x_{1}\right) \mathrm{d} x_{1}\right]\right\rangle \\
& =k_{\mathrm{p}}{ }^{4} \int_{0}^{\infty}\left\langle\lambda(r) \lambda\left(r_{1}\right)\right\rangle G_{\mathrm{p}}\left(x-x_{1}\right)\left\langle P\left(x_{1}\right)\right\rangle \mathrm{d} x_{1},
\end{aligned}
$$

here, use has been made of $\left\langle\lambda(r) \lambda\left(r_{1}\right) P(x)\right\rangle=\left\langle\lambda(r) \lambda\left(r_{1}\right)\right\rangle\left\langle P\left(x_{1}\right)\right\rangle$ as explained in the references on stochastic perturbation theory.

The expression in $\lambda$ becomes, considering (A.1) and (A.3),

$$
\left\langle\lambda(\boldsymbol{r}) \lambda\left(\boldsymbol{r}_{1}\right)\right\rangle=\left\langle\lambda^{2}\right\rangle \Gamma\left(\boldsymbol{r}-\boldsymbol{r}_{1}\right),
$$

where, since the wave is to be taken as plane (in the $x_{2}, x_{3}$ plane)

$$
\Gamma\left(x_{1}, 0,0\right)=\left(1+\frac{x_{1}}{l_{1}}\right) \mathrm{e}^{-x_{1} / l_{1}} .
$$

The $x_{2}$ and $x_{3}$ variables do play a role, however, in expressions such as $\Gamma_{, 3,3}\left(\boldsymbol{r}-\boldsymbol{r}_{1}\right)$ when taking the derivatives; subsequently though we set $x_{2}=x_{2}{ }^{\prime}$ and $x_{3}=x_{3}{ }^{\prime}$ thus the treatment is that of a plane-wave propagating in one dimension although the other dimensions make themselves felt through the $x_{2}$ or $x_{3}$ derivatives of $\Gamma(R)$ which may occur. The treatment, therefore, is not altogether one dimensional.

With the results given here in the appendix the expressions (2.15) and (2.16) can be obtained readily. We do not write them out here, though the formulas $(2.22)$ and (2.23) are their immediate consequences. See Appendix II.

\section{APPENDIX II}

Equations (2.15) and (2.16) are given here with the functions $F$ and $G$ in explicit form.

$$
\begin{gathered}
-K_{\mathrm{p}}{ }^{2}+k_{\mathrm{p}}{ }^{2}=\frac{\left\langle\lambda^{2}\right\rangle{k_{\mathrm{p}}}^{3}}{2 i}\left\{\frac{\left[\alpha_{1}+i\left(K_{\mathrm{p}}-k_{\mathrm{p}}\right)\right]}{\left[\alpha_{1}{ }^{2}+\left(K_{\mathrm{p}}-k_{\mathrm{p}}\right)^{2}\right]}\right\}-\frac{i\left\langle\varepsilon^{2}\right\rangle \alpha_{3}{ }^{2} K_{\mathrm{p}}}{\sigma\left[\alpha_{1}+i\left(K_{\mathrm{p}}-k_{\mathrm{s}}\right)\right]}, \\
-K_{\mathrm{s}}{ }^{2}+k_{\mathrm{s}}{ }^{2}=\frac{\left\langle\lambda^{2}\right\rangle k_{\mathrm{s}}{ }^{3}}{2 i}\left\{\frac{\left[\alpha_{1}+i\left(K_{\mathrm{s}}-k_{\mathrm{s}}\right)\right]}{\left[\alpha_{1}{ }^{2}+\left(K_{\mathrm{s}}-k_{\mathrm{s}}\right)^{2}\right]}-\frac{i\left\langle\varepsilon^{2}\right\rangle \alpha_{3}{ }^{2} K_{\mathrm{s}}}{\sigma\left[\alpha_{1}+i\left(K_{\mathrm{s}}-k_{\mathrm{p}}\right)\right]}\right\} \\
\alpha_{3} \equiv l_{3}{ }^{-1}, \quad \alpha_{1} \equiv l_{1}{ }^{-1} .
\end{gathered}
$$




\section{REFERENCES}

BoURRET, R., Stochastically perturbed systems, with applications to wave propagation in random media, Il Nuovo Cimento, 26, 1-31, 1962.1)

Bourret, R., Propagation of randomly perturbed fields, Can. J. Phys., 40, 782-790, 1962.2)

Bourret, R., Ficton theory of dynamical systems with noisy parameters, Can. J. Phys., 43, 619639, 1965:")

BoURRET, R., Seismic waves in a randomly stratified medium: the parabolic approximation, Part I, submitted to B.S.S.A., 1980.4)

Frisch, U., Wave propagation in random media, in Probability Methods in Applied Mathematics, ed. A. T. Bharucha-Reid, Vol. I, pp. 75-198, Academic Press, N.Y., 1968.5)

Hasan Naymil, Ali., Perturbation Methods, pp. 361-383, John Wiley \& Sons, Inc., N.Y., 1973. ${ }^{07}$

1) A general exposition with especial emphasis on wave problems.

2) The first exposition of method, showing basic examples and showing historical origins.

3) A detailed exposition of the method with higher order approximations and diverse techniques.

4) Internal report, 1980, Venezuelan Foundation for Seismological Research.

B) Excellent overall review, with critical mathematical ovservations.

B) Recent and clear exposition of method and some variations. 